\title{
EL QUIEBRE ÉTICO DE LA COMUNIDAD ÉTICA: LA SOCIEDAD CIVIL ${ }^{1}$
}

\author{
The ethical breakdown of the ethical community: The civil society \\ Patricio Landaeta * \\ Juan Ignacio Arias**
}

\section{RESUMEN}

La presentación de la sociedad civil que se hace en el artículo pretende, por un lado, acabar con la idea ampliamente generalizada de la Filosofía del derecho de Hegel como una simple teleología, donde cada uno de sus momentos orientan su actividad hacia un fin, que es el Estado; y, por otro, en la propia refutación de esta idea, mostrar la actividad negativa de la sociedad civil, como el momento específico del silogismo de la eticidad que escinde sus extremos. Con esto se pretende repensar el ejercicio específico que tiene la sociedad civil dentro del acontecer político, como el momento de quiebre de la unidad estatal y que, por las características que le son propias, determinan la acción del Estado.

Palabras clave: Sociedad civil, Estado, Hegel, eticidad.

\begin{abstract}
The presentation of the civil society made in this paper tries to show two things: on the one hand, it ends with the broadly generalized idea about Hegel's Philosophy of right as

* Centro de Estudios Avanzados, Universidad de Playa Ancha. Valparaíso, Chile. Correo electrónico: patricio.landaeta@upla.cl

** Centro de Estudios Avanzados, Universidad de Playa Ancha. Valparaíso, Chile. Correo electrónico: juan.arias@upla.cl
\end{abstract}

Artículo recibido el 14 de abril de 2014. Aceptado el 11 de noviembre de 2014. 
a simple teleology, where every moment that compose it is oriented toward an end, the state, and, on the other hand, within the refutation of this idea, to show the negative activity as the specific moment of the syllogism that occurs within the civil society that splits both of its extremes. With this in mind we would like to rethink the specific activity that the civil society has inside the political field, as the breaking point of the state's unity, that thanks to its characteristics determine the activity of the latter.

Keywords: Civil society, State, Hegel, ethical life.

${ }^{1}$ La complejidad del término sociedad civil (en alemán bürgerliche Gesellschaft) manteniendo aún una clara contingencia. Si se revisa a partir de una linealidad histórica, esta aparece en el mundo griego, con la salvedad de que en él propiamente no había algo así como una sociedad civil, según se interpreta hoy o según se ha interpretado en la modernidad. Si bien, por lo general, los teóricos políticos han visto en la koinonia politiké aristotélica el origen de la societas civilis latina, tal relación no se puede hacer sino obviando el carácter fundamental sobre el que se asienta la polis griega, como es su calidad de independencia con respecto a otras polis, lo cual conlleva la necesidad de satisfacerse económicamente por sí misma. Esta posibilidad es, por cierto, el rasgo que la diferencia cualitativamente de la sociedad que la precede por naturaleza, como es la familia, pues esta logra satisfacer sus necesidades solo en relación con otras familias. El mundo familiar si bien contiene el elemento privado con el que se ha caracterizado la sociedad civil en distintas instancias, no es algo que se mantenga separado de la comunidad política, muy por el contrario, tiene en esta su posibilidad de subsistencia. La conocida primacía que tiene el Estado por sobre la familia en el mundo griego hace saltar por los aires el vínculo con la sociedad civil, cuestión que alcanza su máxima expresión en el concepto de libertad existente en el mundo griego, donde solo aquel que participaba activamente de los asuntos de la polis (el político propiamente tal), es aquel que gozaba de tal distinción, en tanto que los que no tenían voz para los asunto públicos (aunque sí los tuvieran en la casa, en el dominio privado, como es el caso de la mujer) no eran ciudadanos y, por tanto, tampoco libres.

Ya la definición que da Cicerón de la societas civilis "como un estado jurídico de individuos «vinculados» por «la ley» y por el «derecho común»", expone en su fondo aquel lugar que en la modernidad tendrá. Sin embargo, el momento de conflicto surgirá con el ascenso de la burguesía como destacado agente social, y es eso el conflicto que se desprende desde la sola expresión alemana que sirve para referirse a la sociedad civil: bürgerliche Gesellschaft, que bien podría traducirse, según lo hace José María Ripalda, como sociedad burguesa, cuestión que sonaría por su literalidad de manera correcta. En efecto, como apunta el traductor español, la diferencia se comenzará hacer patente desde el propio Hegel quien introduce en el interior de la sociedad civil los intereses individuales. Eso es correcto, y en este sentido el vínculo con la ley que supone una sociedad civil le viene incorporada por debajo, en vistas a la mejor distribución de aquellos intereses, para que no se produzca un choque entre ellos y para finalmente, no romper con el orden público. Este es, en efecto, el desarrollo que asumirá la sociedad civil, pero en Hegel tal concepción es del todo sesgada, pues si bien ella es la "apariencia exterior" de la eticidad, como esta lesión que se hace de la universalidad, ella no cesa de realizarse en y por esa exterioridad, que la tiene como su fin inmanente. Por ello, realizar la distinción en la sociedad civil, entre civil por un lado y burgués por otro, en Hegel aun no es del todo evidente y conviene mantener esta traducción y así, a la vez, mantener la distancia con el análisis que hará de ella Marx, desde el cual tal distinción de Ripalda sí resulta adecuada. 


\section{INTRODUCCIÓN}

Si bien la sociedad civil tiene una importante y destacada historia en el pensamiento político, no será sino hasta las revoluciones burguesas cuando se convierta en uno de los protagonistas más destacados al interior de la comunidad, y será el filósofo alemán G. W. F. Hegel el primero en reconocer su importancia específica en la modernidad, insertándola al interior de su pensamiento sobre el derecho (en específico, al interior de la comunidad ética o eticidad, Sittlichkeit), cumpliendo una función de quiebre entre las instituciones sociales y políticas clásicas, como son la familia y el Estado. La importancia de esta incorporación radica en la comprensión de la sociedad civil como un momento ético del desarrollo político, asumiendo aquellos contenidos que antes se encontraban marginados, específicamente como aquello otro de los asuntos concernientes a la política propiamente tal. Es así que Hegel comprende dentro de la sociedad civil aquellos elementos que sin ser propiamente universales, establecen mediaciones concretas que orientan las actividades de los particulares hacia la dimensión estatal, pero sin perder la cualidad específica de su particularidad, esto es, sin ejercer una coacción sobre ellos, procurando asegurar de esta manera su libertad.

El pensamiento y esta incorporación de la sociedad civil dentro del pensamiento político hegeliano, ya acontece desde sus escritos de juventud ${ }^{2}$, donde se puede apreciar lo medular de su pensamiento en torno a esta instancia social, al presentarla como aquel momento de escisión al interior de las dos instituciones universales ya señaladas (la familia y el Estado), que es provocada por la propia acción de los particulares (a través del desarrollo de sus propios intereses, caracterizados por el egoísmo y las necesidades individuales) que operan y actúan al interior de lo universal. En el escrito de 1802 (Sobre las maneras de tratar cientificamente el derecho natural) Hegel denominó esta actividad de los particulares (analizándolo desde un imaginario del mundo antiguo) como la tragedia de la ética, para presentar mediante esta figura el desarrollo de las necesidades (naturales, como dijimos, esta es la esfera de los deseos e intereses particulares) que, sin ser universales, son autopuestas por

\footnotetext{
${ }^{2}$ Los estudios que Hegel le dedicara a la sociedad civil (y en específico a la económica, como ciencia que tiene su práctica en este momento de la comunidad) remontan a 1799, cuando leyera el texto de James Stewart: Inquiring into the principles of political economy. Si bien los manuscritos de los apuntes que Hegel hiciera sobre la materia han desaparecido, su discípulo Karl Rosenkranz sí tuvo la oportunidad de leerlo, destacando de ellos: "Se concentraban en él todas las ideas de Hegel relativas a la esencia de la sociedad civil, concernientes a las necesidades y al trabajo, la división del trabajo y los bienes de las diversas clases de la sociedad, concernientes a los pobres y a la policía, los impuestos, etc. Puede encontrarse una síntesis grandiosa sobre la política y la historia... Hegel ataca todo lo que hay de muerto en ese sistema, procurando, en medio de la concurrencia y la mecanización del trabajo, salvar la vida interior del hombre" (Garaudy, 1965: 61).
} 
lo universal para desarrollarse y poder, finalmente, realizarse de un modo concreto, tal como acontecía en la tragedia griega, donde los protagonistas aun yendo contra el destino y esforzándose por cumplir con $s u$ propio y particular fin, desarrollan y le dan vida a la tragedia, para que finalmente el destino se cumpla, aun a costa de ellos ${ }^{3}$. Del mismo modo, la sociedad civil es puesta por la universalidad afuera de sí misma, con fin a reconocerla para poder asumirla posteriormente: "lo viviente a la vez [que] reconoce el derecho de aquello [inorgánico], pone en él mientras tanto lo que sabe [que es] como una parte de sí mismo y lo sacrifica a la muerte, pero simultáneamente se purifica en ello" (Hegel, 1979: 74).

Este sacrificio que realiza lo viviente (el Estado mismo) señala el giro que se encontrará presente en la formulación hegeliana madura de la comunidad ética, ya sin necesidad de recurrir a las figuras del mundo antiguo para pensar su propio tiempo, donde acogiendo los elementos seńalados desarrollará su particular tragedia, y donde su representación no se encuentra determinada por figuras éticas, sino que cada individualidad tendrá sentido solo en la unión con el universal.

Expresado en terminología estrictamente vinculada a la filosofía política, Hegel sostiene:

por ser el estado el espíritu objetivo, el individuo sólo tiene objetividad, verdad y ética si forma parte de él. La unión como tal es ella misma el fin y el contenido verdadero, y la determinación de los individuos es llevar una vida universal. Sus restantes satisfacciones, actividades y modos de comportarse tienen como punto de partida este elemento sustancial y válido universalmente (Hegel, 2004: 228).

Aquello que compone estas determinaciones de los individuos será el contenido de este artículo, pero en su lado formal lo que en el pensamiento de Hegel se mantiene es la presencia del elemento sustancial que es tanto el punto de partida de la sociedad civil, como su fin inmanente. La eticidad en sí misma es una totalidad que comprende cada uno de sus momentos como un desarrollo a partir de sí misma, pero este desarrollo presupone, a la vez, por el carácter lógico que lo determina, la independencia de cada uno de sus miembros, los cuales son para sí independientes del todo. Cada miembro que la compone persigue sus propios fines pero, a la vez, el movimiento de la totalidad tiene como presupuesto la identidad de cada uno de estos miembros, de modo que cuando realicen estos fines, inconscientemente se encontrarán realizando un fin anterior y superior a ellos que les confiere el carácter de ser una unidad ética, sin la cual la comunidad

\footnotetext{
3 "La tragedia consiste en esto, en que la naturaleza ética se separa de sí y, para no implicarse con ella, se opone, como un destino, a su [naturaleza] inorgánica y, mediante el reconocimiento del mismo, se reconcilia en la lucha con la esencia divina como la unidad de ambos" (Hegel,1979: 76).
} 
no sería más que "una acumulación, como una masa carente de forma" (Hegel, 2004: 281), la cual habitaría aquel territorio dominado por la violencia, el miedo y el azar, caracterizado tradicionalmente por la teoría política moderna como estado de naturaleza. Este estado, por el contrario, no es algo allende a la misma política, sino que surge de ella. Es por esto que Hegel rompe con la idea de que aquellos elementos del estado de naturaleza fueran categorías límites de la política, que amenazaban desde un afuera con derrumbar el orden interno. Por el contrario, la amenaza no es externa, sino que late permanentemente en el interior de lo político, y acá aparece ocupando un lugar propio dentro de él: tal lugar es la sociedad civil. Las amenazas pierden su carácter de externas, y se convierten en momentos necesarios para el surgimiento del universal que, en la dimensión objetiva del espíritu, no es otra cosa que el Estado. La movilidad e inquietud que provoca la sociedad civil subvierte el orden político mismo, dando origen a un nuevo modo de entender la política a partir de esta misma inquietud.

\section{EL QUIEBRE DE LA COMUNIDAD ÉTICA: LA SOCIEDAD CIVIL}

En la filosofía de Hegel cada una de las formulaciones en torno las distintas dimensiones que ocupa el espiritu y el saber humano tiene su expresión lógica ${ }^{4}, y$ en este momento específico de la comunidad política, esta expresión manifiesta la circularidad del silogismo en el aparecer de la particularidad (Besonderheit) que rompe con el dominio de lo universal, pasando a ser este su fundamento, pero como forma interna que necesita del desarrollo y despliegue de la particularidad para manifestarse, así como esta tiene la necesidad (Notwendigkeit) de elevarse por sobre sí misma para alcanzar su consistencia en su forma interna ${ }^{5}$.

${ }^{4}$ Cuestión que debe ser entendida de manera estricta: cada uno de los momentos de la filosofía de Hegel se encuentra compuestas de momentos que son necesarios, una necesidad que es lógica. Así, a cada una de las representaciones políticas que rompe con una concepción social existente, le corresponderá una formulación lógica que la fundamente y, específicamente, como corresponde a la sociedad civil, estos serán momentos de la lógica de la esencia, estructura lógica que atraviesa todo el desarrollo de la eticidad, hasta su realidad efectiva. Y así como en ella el ser pierde toda su consistencia cualitativa, del mismo modo la exposición que haremos de la sociedad civil será un ahondamiento en la pérdida de estabilidad de todo el orden político fundamentado en la sustancia ética.

${ }^{5}$ Véase, por ejemplo, el siguiente parágrafo, donde Hegel expresa esta necesidad circular del universal que tiene de la particularidad para desarrollarse (y viceversa): "Todo es concepto, y el existir de éste es la distinción de sus propios momentos, de tal manera que su naturaleza universal se confiere realidad exterior mediante la particularidad y, de este modo y como reflexión negativa hacia sí, se hace singular, o también viceversa, lo real efectivo es un singular que mediante la particularidad se eleva a la universalidad y se hace idéntico consigo mismo" (Hegel, 2008: 260). 
Concretamente, a lo que hacemos referencia es que, junto con estas dos formas de aparecer de lo lógico -y conteniéndolos- acontece el movimiento específico de la Idea al interior de la eticidad, describiendo el paso que se da desde la familia a la sociedad civil, representando el desmantelamiento del universal en una atomización de la familia (Cf. Hegel, 2004: 180-181). Esta rompe su unidad específicamente natural e inmediata, amparada en el amor y la confianza, para convertirse en muchas familias, entendidas cada una como particularidades que lucharán y velarán por sus propias necesidades, no siendo una unidad sustancial entre todas, sino individualidades que dispondrán cada cual para sí mismas de lo que necesitan con fin de lograr su satisfacción. De esta manera cada una de estas particularidades perderá su contenido ético comunitario, replegándose este hacia su interioridad, como conciencia moral. La familia, como en Aristóteles, continúa siendo la base de la comunidad, pues puede ser el origen natural de un pueblo, realizándose este a partir de una ampliación de ella; o bien como una reunión de familias dispersas, que son unidas por coerción o por la unión voluntaria de ellas, en vista a la satisfacción de sus necesidades. Sin embargo, esta última forma de reunión ya ha perdido su contenido ético, pues su principio no es la unidad sustancial, sino la satisfacción de las necesidades de distinta índole, al punto que Hegel sostiene que en este proceso de desmantelamiento de la familia, la eticidad parece perdida, "pues para la conciencia la identidad de la familia es lo auténticamente primero, divino y que impone deberes" (Hegel, 2004: 181). En cambio lo que se ofrece a la vista del espectador es el extraño espectáculo, propio de la época moderna (tal como sostiene Hegel: "La concepción de la sociedad civil pertenece por otra parte al mundo moderno" (2004: 183), de la total dispersión fragmentaria de particularidades, cada cual afanadas en conseguir un punto de consistencia que le asegure la existencia en los distintos elementos que constituyen a la sociedad civil, en las formas de estabilidad económica, mediante la organización del trabajo, la "protección de la propiedad" y "la prevención contra la contingencia", mediante la administración de la justicia, y "el cuidado de los intereses particulares" (Hegel, 2004: 187-188) gracias a la acción de la policía.

La ruptura que se da entre las dos formas universales de aparecer del mundo ético, hace que se desfonden la primera en la otra (la familia se diluye en lo particular), y el Estado que lo contiene, se escinde al interior de sí. Cada uno de los momentos que componen el silogismo de la eticidad son asumidos en los tres organismos que constituye el mundo social (Recuérdese: familia - sociedad civil - Estado).

En una esclarecedora nota ubicada en el mecanismo al interior de la lógica de la Enciclopedia, Hegel expone de manera general la interna relación que existe entre los tres momentos, resaltando la común dependencia que se da entre ellos y la satisfacción que encuentra cada nueva figura. 
El Estado en el campo de lo práctico es un sistema de tres silogismos. 1) El singular (la persona) se concluye mediante su particularidad (las necesidades físicas y espirituales, [que es aquello] que más configurado da [lugar a] la sociedad civil), con lo universal (la sociedad, el derecho, ley, gobierno). 2) La voluntad y actividad de los individuos es [también] lo mediador que da satisfacción a las necesidades en la sociedad, en el derecho, etc., del mismo modo que da cumplimiento y realización efectiva a la sociedad, al derecho, etc. 3) Pero [también] lo universal (Estado, gobierno, derecho) es el medio sustantivo en el que los individuos y su satisfacción tienen y mantienen su realidad plena, su mediación y su subsistencia. Cada una de las determinaciones, en tanto la mediación la concluye con el otro extremo, se concluye asimismo consigo, se produce, y esta producción es autoconservación. -Sólo mediante la naturaleza de este concluir, o sea, mediante esta triplicidad de silogismos con los mismos términos, es como verdaderamente se comprende un todo con su organización (Hegel, 2008: 274-275)'.

El movimiento es propio del silogismo de la existencia (por ello aunque revele un interno movimiento de las figuras que lo componen, no será conclusivo, pasando el término medio a desfondarse en el silogismo de la reflexión).

Siguiendo la cita, el primer silogismo presenta la siguiente figura: $S$ (singular) - P (particular) - U (universal), donde P, que en cuanto organismo es la sociedad civil, establece un vínculo entre el término mayor (el Estado) aún abstracto y el término menor (la persona o familia) aun meramente singular, contingente. Esto quiere decir que el singular -la persona, la familia- satisface sus necesidades mediante la intervención de la sociedad civil y al hacerlo, por ejemplo, a través del trabajo, a la vez que se satisface, desarrolla el universal, el propio Estado se ve beneficiado mediante su trabajo. Por tanto, lo particular satisface las necesidades de cada uno al mediar como vínculo entre el uno y otro, despojándolos de sus primeras características unilaterales (tal es la presentación que se hace en la eticidad de la Filosofía del derecho).

La segunda figura (donde el término mayor deviene menor, el menor, medio, y el término medio, mayor, quedando formalmente expresado como $\mathrm{U}$ - S - P) establece su centro en la persona que mediante su actividad vitaliza los momentos abstractos de los organismos, los que pierden su calidad de abstractos y los inserta en la existencia. De esta manera, el Estado tiene que expresar la vida íntima de los pueblos (a través de la función legislativa), con fin a que la sociedad

${ }^{6}$ Esta nota es analizada con particular claridad por Ramón Valls Plana en el artículo "Somos conflictivos, pero... Actualidad de la tesis de Kant sobre la insociable sociabilidad de los humanos y su prolongación por parte de Hegel", artículo en coautoría con Ferrán Requejo. 
civil se ajuste a las normas establecidas por él, dado a que se da un ajuste pleno entre las leyes y ella misma ${ }^{7}$.

De modo que así puede surgir la tercera forma $(\mathrm{P}-\mathrm{U}-\mathrm{S})$ que pone como medio el universal que ya se ha establecido como realidad efectiva y resplandece como la verdad de los extremos, siendo ahora, como medio, universal concreto. En este tercer silogismo, la sociedad civil se ajusta a los requerimientos y necesidades de las personas, dado que estas necesidades ya no son meras menesterosidades (Bedürfnis) sino que coinciden con la necesidad del universal (y, por lo tanto, esta necesidad ahora se entiende en su dimensión de necesidad lógica: Notwendigkeit).

Explicada de esta manera general la circularidad que se da en la eticidad, se logra ver cómo se abre paso la realización del singular (individuo) en el universal (Estado). De esta manera, captando la fluida solidez del silogismo, se puede comprender que: "Esta unidad sustancial es el absoluto e inmóvil fin último en el que la libertad alcanza su derecho supremo, por lo que este fin último tiene un derecho superior al individuo, cuyo supremo deber es ser miembro del estado" (Hegel, 2004: 227). Cuestión que debe entenderse dentro del proceso señalado: la unidad sustancial es la unidad de los momentos del silogismo funcionando al interior del Estado, por ende cada figura de los recién señalados tres silogismos tienen "la necesidad de que cada momento, en cuanto determinación del concepto mismo, devenga igualmente el todo y el fundamento mediador" (Hegel, 2008: 264). De modo que el movimiento que realiza el singular, el particular y el universal (vistos desde los organismos que constituyen la eticidad, como familia, sociedad civil y Estado, respectivamente) supone una interacción con fin a satisfacer las necesidades impuestas por sus extremos. En cuanto ellos cumplan la función de concluirlos, cada uno se realizará de manera efectiva en el otro, teniendo en su otro su plenitud y acabamiento. Cada momento del tripe silogismo satisface la necesidad de los extremos, realiza a los otros, se dona en ellos, y en esta donación es como se cumple a sí mismo. . Aquel "supremo deber" que es "ser miembro del

\footnotetext{
${ }^{7}$ Recuerde que desde temprano (y a lo largo de toda su teoría del derecho) la concepción que tiene Hegel de la constitución, lejana a un código positivo de leyes, se identifica con la vida ética de los pueblos. "Puesto que el espíritu sólo es efectivamente real en el modo que se sabe, y el estado, en cuanto espíritu de un pueblo, es al mismo tiempo la ley que penetra todas sus relaciones, las costumbres y la conciencia de los individuos, la constitución de un pueblo determinado depende del modo y de la cultura de su autoconciencia. En ella reside su libertad su libertad subjetiva y en consecuencia la realidad de la constitución” (Hegel, 2004: 257).

${ }^{8}$ De esta manera se ve que la coerción totalitaria que ejerce el Estado sobre el sujeto, que se le achaca por lo general a Hegel, es solo una mirada sesgada sobre la dimensión global en los que ambos se encuentran. El problema de la coerción es el problema con que Hegel se tiene que enfrentar, el que supone un drama mayor (o al menos más sutil) al problema político de la coerción estatal, sin embargo, desde ya se puede sostener que Hegel se encuentra muy lejos (doctrinariamente) de considerar la coerción como un modelo válido a seguir, muy por el contrario, desplegará hasta extremos
} 
estado", porque este como "fin último tiene un derecho superior al individuo", es verdad solo en el sentido de que en éste el individuo se realiza plenamente, lo cual en última instancia es lo mismo que alcanzar una satisfacción plena, cuestión perseguida por todo organismo que es constituido por la carencia, fondo último natural que se mantiene presente en el desarrollo del espíritu.

Sin embargo, si el Estado es el "fin último" donde los demás se concluyen, se presenta un momento de desequilibrio en la estructura interna del silogismo, pues cada uno de estos intentos de aproximación a lo otro, una vez que se ha realizado el vínculo y pareciera darse una permanencia en la unidad de los extremos, alcanzando la finalidad su cumplimiento, se vuelve abrir, rompiéndose la unidad en múltiples puntos expandidos en su interior, pues el Estado lejos se encuentra de asumir la clasificación de ser algo en sí mismo plenamente racional, ni dentro del sistema ni de manera contingente", sino que, en palabras de Hegel, "el estado debe considerarse como un gran edificio arquitectónico, como un jeroglífico de la razón, que se expone en el mundo" (Hegel, 2004: 264). El Estado no puede constituirse como racional en sí mismo, sino como jeroglífico de la razón, puesto que se encuentra atravesado por el "arbitrio la contingencia y el error" (Hegel, 2004: 232), cuestión que lo constituye estructuralmente. De modo que la cerrazón que él otorga se mueve dentro de su propia esfera, la cual se perpetúa dentro de su movimiento, pero que no puede clausurarse en sí mismo, sino que va abriendo grietas a través y por esa contingencia y arbitrariedad, la cual es siempre exterior.

Por ello, si la unidad sustancial que proporciona el Estado es el "fin último" para la realización de la libertad, al interior de esa unidad acontece el movimiento que se da en la teleología de la lógica, en el minuto exacto antes que esta pase a la Idea, según lo expresa Hegel: "el fin llevado a cabo es algo tan roto en sí mismo como lo era el término medio y el fin inicial. [...] El fin alcanzado es por ello solamente un OBJETO que es a la vez medio o material para otro fin y así sucesivamente hacia el infinito" (Hegel, 2008: 282).

que no puede seguir mediaciones al interior de las instituciones para que mediante un movimiento esencial se manifieste lo universal, sin necesidad de sometimiento alguno. A nuestro entender, el único momento en que se encuentra validado el uso del poder (Gewalt) es en el momento histórico de la fundación de las ciudades, como imposición del derecho, una vez instaurado este, tal uso del poder queda marginado del gobierno político. Lo que queda por investigar es si ese momento es tan solo un momento histórico o es un momento político de suyo, el que no se reduce a una etapa del pasado, sino que surge (y en este surgimiento, guarda una potencialidad siempre latente) cada vez que el derecho queda marginado del gobierno político.

${ }^{9}$ Como ciertos grupos sostuvieron que hacia Hegel, a raíz de la célebre sentencia del Prefacio de la Filosofía del derecho, que identificaba lo racional con lo real, sosteniendo que aquello racional era el estado prusiano. En cambio, Hegel se encuentra lejos de clasificar no solo un momento histórico como racional y como supuesto fin cumplido de la historia toda, donde esta se vería cumplida, clausurándose en sí misma. 
Según esto, en el momento conclusivo que articula lo universal, se produce una caída, la cual desfonda cualquier tipo de cerrazón que se pretenda establecer producto de la actividad orientada a un fin. Este permanece como exterioridad, y esta permanencia es lo que hace que la caída, el desfondamiento que se produce en la tendencia al fin no sea un hundimiento hacia adentro, hacia la subjetividad, sino que es una caída hacia fuera, hacia lo objetivo. En este abismarse en la exterioridad lo que se hace es crear nuevos contenidos que van posibilitando nuevos niveles de simbolización con el fin de acercar los lazos de los extremos que persisten en su unilateralidad, intentando estrechar un espacio imposible de ser llenado. Y es en esta imposibilidad de alcanzarse, que cada uno de los esfuerzos que han pretendido lograrlo, no han hecho sino emerger como niveles históricos o niveles políticos. Esta caída se vuelve inmediatamente actividad sobre el mundo, y es el momento reflexivo al interior de cada uno de los desarrollos de estos niveles.

Los tres silogismos al interior de la eticidad que acabamos de analizar, no son sino la construcción conceptual de ella, permaneciendo en las sombras lo que podría ser su lado oculto, aunque en verdad es lo que se hace manifiesto de mayor manera, como es su aparición fenoménica, la cual acoge la realización en el tiempo de cada uno de los momentos que hemos visto.

Todo esto nos lleva a establecer un momento de inestabilidad en aquella pretendida paz y satisfacción alcanzada tras la circularidad de los tres silogismos, volviendo a relucir la incesante insatisfacción que se destaca en la sociedad civil, que hunde al Estado en esta inestabilidad. Esto, dado que el mismo silogismo tiende a hundirse en la insatisfacción, pues su movimiento tiene como contenido la actividad de la particularidad, que tienen como sello distintivo las pasiones individuales, que se encuentran a la base de cada relación intersubjetiva, sucediendo en ese silogismo algo semejante a lo que acontece la clausura de la teleología, que al momento de cerrarse, abruptamente se desgarra en su elemento externo ${ }^{10}$. Tal como señala el filósofo español Félix Duque, citando a Hegel: "Si el [fin final] estuviera realizado, si el sujeto fuera ético, caería entonces la subjetividad, el bien valdría como por naturaleza y la libertad del sujeto desaparecería: el sujeto sólo es libre en la lucha" (Duque, 1987:139).

Si bien en los tres silogismos se alcanzó una realización circular y plena, esta se da solo como idea pues, antes de ello, el acontecer fenoménico pone trabas a la construcción, en su momento más alto, en el Estado, ya que este último peldaño de la sociedad no puede renunciar a aquello que lo constituye, como es la referida acción de la particularidad que le proporcionan una realización concreta.

10 "Ha cobrado estado solamente como forma extrínsecamente puesta en el material previamente hallado, forma que en virtud del limitado contenido finalístico es igualmente una determinación contingente" (Hegel, 2008: 282). 
El estado -dice Hegel- no es un producto artificial sino que se haya en el mundo, y está por lo tanto en la esfera del arbitrio la contingencia y el error por lo cual un mal comportamiento puede desfigurarlo en muchos aspectos. Pero incluso el hombre más despreciable, el delincuente, aunque sea un enfermo o un inválido, no por eso deja de ser un hombre viviente; lo afirmativo, la vida, existe a pesar de las carencias. De este aspecto afirmativo es de lo que aquí se trata (Hegel, 2004: 232).

Y cabría agregar, lo que ya Hegel dice en otros lugares, que la vida no sólo existe a pesar de esas carencias, sino en y por esas carencias conoce su infinitud ${ }^{11}$. La persistencia de estas no desaparece y es justamente a través de ellas que el Estado no logra cerrarse, pero son también esas carencias lo que le dan vida y posibilitan su movimiento.

Es precisamente esta incorporación de aquello otro (la contingencia, el arbitrio, lo natural) que presupone lo político será en buena medida la importancia que tendrá la concepción política posthegeliana, al caracterizarse la sociedad civil a partir de las revoluciones burguesas como el espacio específico del desarrollo de las libertades, distinguiéndose en cada una de las presentaciones que de ella se hagan el valor negativo o positivo que tenga ese desarrollo, la actividad negativa o positiva que debe ejercer sobre el Estado (refiriéndonos con negativo o positivo no a un dominio axiológico sino, como lo hemos destacado en el pensamiento de Hegel, dinámico de la política, esto es, la función que desempeña la sociedad civil en relación a las distintas esferas que componen la comunidad ética).

De este modo, como bien destaca el jurista italiano Norberto Bobbio, la concepción que se tenga de la sociedad civil siempre se encuentra en relación con el Estado (como aquello otro de este) y, dentro de esta relación, se pueden distinguir varías acepciones: "lo no-estatal con lo pre-estatal, con lo anti-estatal o con los post-estatal” (Bobbio, 2009: 41). Cada una de estas acepciones han tenido una formulación política determinada, pero comprensivamente se puede entender lo pre-estatal como el dominio que, sin ser propio del Estado, determina su desarrollo, en cuanto es ahí donde surgirían y se establecería las condiciones materiales que condicionarían su posterior funcionamiento. La acepción anti-estatal, siguiendo la línea de lo dicho, se caracterizaría por ser dinámicamente negativa (aunque, en sí misma -como apunta Bobbio- tendría un valor positivo). Dicho de otro modo: acá lo anti-estatal no se expresa bajo la idea del fin o acabamiento del Estado (como acontece con lo post-estatal) sino como osificación de la sociedad civil en tanto

11 "Sólo un viviente siente carencias, puesto que sólo en la naturaleza del concepto está que él sea la unidad de sí mismo y de su opuesto determinado. Donde hay una limitación, ésta es una negación sólo para un tercero, para una comparación exterior. Pero carencia lo es en tanto se da en uno solo el estar más allá, la contradicción como tal es inmanente y está puesta en él. Un tal capaz de tener en sí mismo la contradicción de sí y soportarla es el sujeto; eso constituye su infinitud”'(Hegel, 2008: 415). 
estamento independiente y con leyes propias (nunca mejor dicho, aquí es donde se jugaría y se pediría la independencia de las leyes del mercado, tal como lo hace el liberalismo): es la expresión más propia de la separación de lo privado en relación a lo público, donde se pide (y exige) la delimitación específica del funcionamiento de la sociedad civil. Por último, la concepción post-estatal presenta una dimensión dinámica positiva del Estado, en cuando se serviría de él, para acabarlo, pero en un acabamiento que surgiría, se podría decir, de manera implosiva, en cuanto los mecanismos políticos de los que se sirve el Estado, una vez alcanzado y satisfechas las condiciones y necesidades sociales, perdería su función, alcanzándose y agotándose en ese final.

Tales caracterizaciones comprenden y, de alguna manera, parten de la presentación hecha previamente de Hegel (algunas en completa dependencia con ella y otras como parte del desarrollo histórico de la idea de sociedad civil), sin embargo, la primera de ellas, la relación de la sociedad civil como una esfera preestatal, es la que a nuestro parecer ofrece a su concepto un valor político ajustado a nuestros tiempos y es la que se ha expresado en la formulación hegeliana, como un momento independiente de la comunidad, pero que en la fluidez negativa de su esfera, propicia la actividad de las otras ${ }^{12}$. De esta manera, se establecen vínculos de dependencia justamente por las limitaciones patentes que existen en la sociedad civil, centrada en la acción egoísta de los individuos; tal división mantiene las diferencias, pero a la vez las reúne con fin a satisfacer las necesidades de aquella fragmentada reunión. Es, por tanto, en el mutuo movimiento, tanto de la sociedad como del Estado, donde se alcanza el contenido desarrollado por el hombre en el proceso social, revelándose en tal movimiento una práctica concreta del hombre como parte de la realidad política.

\footnotetext{
${ }^{12}$ Tal es la concepción del joven Marx en su crítica a la filosofía del estado de Hegel: "Familia y sociedad burguesa se presentan como el oscuro fondo natural del que emerge la luz estatal. El material de Estado serían sus asuntos -familia y sociedad burguesa-, en cuanto son partes constitutivas del Estado, que participan en él como tal"(Marx, 2002: 70).

De esta manera el punto focal de la crítica hecha por Marx es propiamente por la forma de unión que pueda proporcionar el Estado político, pues tanto Hegel como Marx se encuentran de acuerdo sobre la separación entre los componentes de la comunidad. Sin embargo, el elemento vinculante para Hegel se daría por la articulación de lo particular por lo general, en tanto que Marx apela a la formula inversa: de la sociedad ha de surgir el Estado político, cuestión que implica que el mismo principio que gobierna y es el fin del Estado hegeliano (la libertad), vuelve a sufrir una inversión en la fórmula de Marx, donde ya no es la libertad el centro de la política, sino la emancipación.
} 


\section{CONCLUSIÓN}

El presente artículo ha mostrado una apertura radical, pues el objeto propio de su estudio se encuentra imposibilitado de cerrarse. Cualquier pretendido cierre que se dé por el lado fenoménico ha de venir desde un afuera, como violencia externa, siendo más bien reclusión. La fuerza que contiene el impulso orientado a un fin en cuanto se encuentra en vínculo con esta realidad finita, abisma su final conclusión antes de aparecer como idea. Se rompe en su interior y persiste en la exteriorización, donde acontece la existencia. Esta es la historia que se sigue escribiendo de la sociedad civil, la que señala la imposibilidad de su acabamiento y clausura en lo estatal, y la que posibilita, a la vez, cada nuevo emerger y la que marca un compromiso con aquello que justamente la imposibilita, pues es con ello con lo que, al fin y al cabo, se realiza en cada momento.

Hegel, en este sentido, no solo ha leído bien su tiempo al introducir el contenido natural que inquieta desde su centro a la sociedad civil (graficado claramente al insertar la economía política en el centro de la teoría de la eticidad), con ello, además, nos entrega herramientas con las que podemos interpretar el nuestro, a partir de un movimiento interno que contenga el conflicto político, pues en sí misma la sociedad civil es una figura conflictiva, por incubar en su seno un cruce que el pensamiento político tradicional había sabido mantener a raya, cada cual dentro de su propia esfera, como es la de lo público y lo privado.

\section{REFERENCIAS}

Bobbio, Norberto. Estado, Gobierno y Sociedad. Por una teoría general de la politica. México: FCE, 2009.

Duque, Félix. Indigencia de la necesidad. En Eticidad y Estado en el Idealismo alemán. Valencia: Natan Estudios, 1987.

Garaudy, Roger. Dios ha muerto. Un estudio sobre Hegel. Buenos Aires: Editorial Platina, 1965.

Hegel, Georg Wilhelm Friedrich. Enciclopedia de las ciencias filosóficas. Trad. Ramón Valls Plana. Madrid: Alianza Editorial, 2008.

Hegel, Georg Wilhelm Friedrich. Principios de la filosofia del derecho. Trad. Juan Luis Vermal. Buenos Aires: Editorial Sudamericana, 2004. 
Hegel, Georg Wilhelm Friedrich. Sobre las maneras de tratar cientificamente el derecho natural. Trad. Dalmacio Negro Pavón. Madrid: Aguilar, 1979.

Marx, Karl. Crítica de la Filosofía del Estado de Hegel. Madrid: Biblioteca Nueva, 2002.

Valls Plana, Ramón y Requejo Coll, Ferrán. "Somos conflictivos, pero... Actualidad de la tesis de Kant sobre la insociable sociabilidad de los humanos y su prolongación por parte de Hegel”, Isegoría 37 (2007): 127-163. 